\title{
Monopoles and Rational Maps: A Note on a Theorem of Donaldson
}

\section{Jacques Hurtubise}

Département de Mathématiques, UQAM, C.P. 8888, Succursale "A", Montréal, Québec, Canada, H3C 3P8

\begin{abstract}
In a recent paper, Donaldson gave a description of the moduli space of SU(2) monopoles in terms of rational maps; this was done indirectly, via the associated solution of Nahm's equations. We give here an interpretation of these rational maps in terms of the monopole's spectral curve, and then as "scattering data" for the monopole itself.
\end{abstract}

\section{Introduction}

In [1], Donaldson, in proving a conjecture of Atiyah and Murray, gave a description of the moduli space $M_{k}$ of $\mathrm{SU}(2)$-monopoles (solutions to the $\mathrm{SU}(2)$ Bogomolny equations) of charge $k$, showing that the space $R_{k}$ of rational maps $f: \mathbb{P}_{1}(C) \rightarrow \mathbb{P}_{1}(C)$ of degree $k$, such that $f(\infty)=0$, could be identified with a circle bundle over $M_{k}$. The construction is done indirectly, using the fact $[3,4]$ that monopoles can be obtained, via an infinite dimensional ADHM construction, from solutions to the Nahm equations, i.e. $k \times k$ matrix valued functions $T_{i}(s), i=1,2,3, s \in(0,2)$ such that

$$
\begin{gathered}
\frac{d T_{i}}{d s}+\frac{1}{2} \Sigma \varepsilon_{i j k}\left[T_{j}, T_{k}\right]=0, \\
T_{i}^{*}(s)=-T_{i}(s), \\
T_{i}(2-s)=T_{i}(s)^{T},
\end{gathered}
$$

The $T^{i}$ are analytic over $(0,2)$, with simple poles at 0,2 .

The residues of $T_{i}$ at $s=0$ form an irreducible representation of su(2).

To each $O(k, \mathbb{C})$ equivalence class of such solutions, Donaldson associates a circle of rational maps. 
On the other hand, in $[2,3]$, Hitchin showed how one could associate to each monopole a spectral curve $S$ in $T \mathbb{P}_{1}(\mathbb{C})$; he then showed how one could obtain solutions to the Nahm equations in terms of flows on the Jacobian of $S$.

The purpose of this note is to translate the rational maps of Donaldson into the domain of spectral curves, linking these two algebro-geometric invariants associated to a monopole. The rational maps are then interpreted directly in terms of monopoles, and are found to be constructed from "scattering data" associated to a set of $k$ parallel lines corresponding to points on $S$; Donaldson's result then implies that each such set of data determines a monopole.

\section{The Rational Map}

We first recall how Donaldson constructs a rational map from a solution to (1)-(5). Set $A_{0}=T_{1}+i T_{2}, A_{1}=-2 i T_{3}, A_{2}=T_{1}-i T_{2}$; one has:

$$
\frac{d A_{0}}{d s}-\frac{1}{2}\left[A_{1}, A_{0}\right]=0 \text {. }
$$

From (5), one finds that the residue of $A_{1}$ at $s=0$ has eigenvalues $-(k-1)$, $-(k-3), \ldots,(k-1)$; let $p$ be an eigenvector of norm 1 associated to the eigenvalue $(k-1)$. Now consider maps $v:(0,2) \rightarrow \mathbb{C}^{k}$ such that:

$$
\frac{d v}{d s}-\frac{1}{2} A_{1} v=0
$$

There is a unique solution $u$ to (7) such that $s^{-(k-1) / 2} u \rightarrow p$ as $s \rightarrow 0$. Set $B=-A_{0}(1), W=u(1)$; then

$$
f(z)=W^{T}(z I-B)^{-1} W
$$

defines the rational map.

We remark in passing that, if one considers $\mathbb{C}^{k} \times(0,2)$ as a vector bundle $E$ over $(0,2)$, and the $A_{i}$ as sections of End $(E)$, then, with respect to a trivialisation of $E$ by solutions of (7), one has from (6) that $A_{0}$ is a constant.

\section{Nahm Equations and Spectral Curves}

We use the notation of [3]. Cover $\mathbb{P}_{1}(\mathbb{C})$ by two standard affine sets $U_{0}$, $U_{1}$, and let $\zeta^{\prime}$ be a coordinate on $U_{0}$; then $T \mathbb{P}_{1}(\mathbb{C})$ has local coordinates:

$\left.(\eta, \zeta) \rightarrow \eta \frac{d}{d \zeta^{\prime}}\right|_{\zeta^{\prime}=\zeta} . T \mathbb{P}_{1}(\mathbb{C})$ has a real structure $\tau$, given by $\tau(\eta, \zeta)=\left(-\bar{\eta} / \zeta^{2},-1 / \bar{\zeta}\right)$. Also, setting $\pi: \quad T \mathbb{P}_{1}(\mathbb{C}) \rightarrow \mathbb{P}_{1}(\mathbb{C})$ the projection, $\tilde{U}_{i}=\pi^{-1}\left(U_{i}\right)$, then, over $T \mathbb{P}_{1}(\mathbb{C})$, one has line bundles $L^{s}, s \in \mathbb{C}$, and $\mathcal{O}(n), n \in \mathbb{Z}$, whose transition functions from $\tilde{U}_{1}$ to $\tilde{U}_{0}$ are $\exp (s \eta / \zeta)$ and $\zeta^{n}$, respectively. Set $L^{s}(n)=$ $L^{s} \otimes \mathcal{O}(n)$.

To each monopole of charge $k$, Hitchin [2], associates a spectral curve $S$ of 
genus $(k-1)^{2}$ in $T \mathbb{P}_{1}(\mathbb{C})$, with equation

$$
\eta^{k}+a_{1}(\zeta) \eta^{k-1}+\cdots+a_{k}(\zeta)=0
$$

where $a_{i}$ is a polynomial of degree $2 i$. One has that $S$ is real ( $\tau$-invariant), that $L^{2}$ is trivial over $S$, and that $H^{0}\left(S, L^{s}(k-2)\right)=0$ for $s \in(0,2)$.

Solutions to Nahm's equations are described as follows: ([3], pp. 165-171) first, one defines a rank $k$ bundle $E$ over $(0,2)$ by setting, for $s \in(0,2)$,

$$
E_{s}=H^{0}\left(S, L^{s}(k-1)\right) \text {. }
$$

One then obtains well defined sections $\tilde{A}_{i}$ of End $(E)$ by demanding, for all $v \in E_{s}$,

$$
\left(\eta+\tilde{A}_{0}+\zeta \tilde{A}_{1}+\zeta^{2} \tilde{A}_{2}\right) v=0 .
$$

To turn the $\tilde{A}_{i}$ into matrices $A_{i}$, one must trivialise $E_{s}$. One does this by choosing a basis of sections $t=\left(t_{1}, \ldots, t_{k}\right)$ of $E$ such that, writing $t$ over $\tilde{U}_{0}, \tilde{U}_{1}$ as $h_{0}(s, \eta, \zeta)$, $h_{1}(s, \eta, \zeta)$ with $h_{0}=\exp (s \eta / \zeta) \zeta^{k-1} h_{1}$, one has, with respect to this basis:

$$
\frac{d h_{0}}{d s}+h_{0}\left(\frac{1}{2} A_{1}+\zeta A_{2}\right)=0 .
$$

(Hitchin's $A_{i}$ are the transpose of ours.) One can then show that one has a solution to (1), (4), (5). To get conditions (2) and (3), Hitchin defines a hermitian and a real structure on $E: \tau$ lifts over $S$ to an antilinear map $\sigma: H^{0}\left(S, L^{s}(k-1)\right) \rightarrow$ $H^{0}\left(S, L^{-s}(k-1)\right)$. Thus, if $u, v \in E_{s}, u \sigma(v) \in H^{0}(S, \mathcal{O}(2 k-2))$. However, such an element can be expanded uniquely as $C_{0} \eta^{k-1}+C_{1}(\zeta) \eta^{k-2}+\cdots+C_{k}(\zeta)$, with $C_{0}$ a constant. One defines the inner product:

$$
\langle u, v\rangle=C_{0} .
$$

More explicitly, representing, as above, $u$ by functions $f_{0}, f_{1}$, and $v$ by $g_{0}, g_{1}$, one has:

$$
\langle u, v\rangle=\eta^{k-1} \text { - term of } f_{0}\left(g_{1} \circ \tau\right) .
$$

This defines the hermitian structure; for the real structure, let $a$ be a section of $L^{2}$ on $S$. Then $a \cdot \sigma(a)$ is a constant, which can be set to one ([3], p. 181). (Thus $a$ is defined up to a constant of norm one; as we will see later on, this gives the circle ambiguity in defining the rational map.)

One defines the real structure:

$$
\sigma^{\prime}: H^{0}\left(S, L^{s}(k-1)\right) \rightarrow H^{0}\left(S, L^{2-s}(k-1)\right)
$$

by $\sigma^{\prime}=a \sigma$. At $s=1$, one has an orthogonal structure:

$$
(u, v)=\left\langle u, \sigma^{\prime} v\right\rangle=\eta^{k-1} \text {-term of }(\sigma(a) u v) .
$$

We are now ready to translate the rational map into spectral curve terms. We begin by noting that the trivialisation of sections $v(s)$ of $E$ satisfying (7), is given by writing $v(s)$ as $g_{j}(s, \eta, \zeta)$ over $U_{j}, g_{0}(s, \eta, \zeta)=\exp (s \eta / \zeta) g_{1}(s, \eta, \zeta)$, and demanding that $g_{0}(s, \eta, 0)$ be constant in $s$ : for this, one refers to $(12)$. Let $\left(\eta_{i}, 0\right), i=1, \ldots, k$ be the points of intersection of $S$ with $\zeta=0$ which, for the moment, we suppose distinct. 
As $H^{0}\left(S, L^{s}(k-2)\right)=0$, one can choose a basis $v_{i}(s)$ of $E(s)$ with corresponding representative functions $g_{j i}(s, \eta, \zeta)$ over $\tilde{U}_{j}$ such that:

$$
g_{0 i}\left(s, \eta_{j}, 0\right)=\delta_{i j}
$$

We will compute the rational map with respect to this basis; first, the ingredients:

The matrix $B$ : From (11), one has that $A_{0}(s)=-\operatorname{diag}\left(\eta_{i}\right)$ : therefore, $B=\operatorname{diag}\left(\eta_{i}\right)$.

The vector $W$ : One begins by noting that there are three different possible types of trivialisations of $E$, as one approaches $s=0$ :

1) by sections $r_{i}(s)$, which tend continuously to non-zero sections $r_{i}(0) \in$ $H^{0}(S, \mathcal{O}(k-1))$,

2) by sections $t_{i}(s)$, satisfying (12) (a "Nahm" trivialisation),

3 ) by sections $v_{i}(s)$, satisfying (7), with fixed "values" at $\zeta=0$.

From [3], p. 179, one can choose $r_{i}$ so that $r_{i}=s^{(k-1) / 2} t_{i}$. On the other hand, referring to [1], the section $u$ that one wants (a constant linear combination of the $v_{i}$ ) satisfies $u(s)=s^{(k-1) / 2} \Sigma a_{i}(s) t_{i}(s)$ with $a_{i}(0)$ finite, not all zero. Thus, $u(0)=\Sigma a_{i}(0) r_{i}(0)$; however, the $r_{i}(0)$, as sections of $H^{0}(S, \mathcal{O}(k-1))$, are all lifted by $\pi^{*}$ from $\mathbb{P}_{1}(\mathbb{C})$ : see [3], Prop. 5.1. Thus, the values of $u(0)$ at the $\left(\zeta_{i}, 0\right)$ are all the same. The same is then true for $u(1)$; in the $v_{i}$-trivialisation,

$$
u(1)=C \cdot(1,1, \ldots, 1) .
$$

There remains the problem of computing $C$. One wants $u(s)$ such that $s^{-(k-1)}\langle u(s), u(s)\rangle \rightarrow 1$ as $s \rightarrow 0$. Referring to (14), now note the fact that $f_{0}(s, \eta, \zeta), f_{1}(s, \eta, \zeta)$ represent a section $u$ of $L^{s}(k-1)$ over $S$ iff $f_{0}(s, \eta, \zeta)=$ $f_{0}\left(s, s^{-1} \eta, \zeta\right), f_{1}(a, \eta, \zeta)=f_{1}\left(s, s^{-1} \eta, \zeta\right)$ represent a section $\tilde{u}$ of $L(k-1)$ over $s \dot{S}$, where $s$ has equation:

$$
\eta^{k}+s a_{1}(\zeta) \eta^{k-1}+\cdots+s^{k} a_{k}(\zeta)=0 .
$$

Now $\langle u, u\rangle=\left(\eta^{k-1}\right)$-term of $f_{0}\left(f_{1} \circ \tau\right)=s^{k-1} .\left(\eta^{k-1}\right)$-term of $\tilde{f}_{0} \cdot\left(\tilde{f}_{1} \circ \tau\right)$; one thus wants $\tilde{u}(s)$ such that the $\left(\eta^{k-1}\right)$-term of $\tilde{f}_{0} \cdot\left(\widetilde{f}_{1} \circ \tau\right)$ is 1 at $s=0$. At $s=0$, $\widetilde{f}_{0}, \widetilde{f}_{1}$ represent a section of $L(k-1)$ over the $k^{\text {th }}$ formal neighbourhood $\left(\eta^{k}=0\right)$ of $(\eta=0)$. One has, from [3], p. 178, that, over $\eta=0, f_{0}=C, f_{1}=C \zeta^{k-1}$; from [3, Lemma 5.4], one infers, that, over $\left(\eta^{k}=0\right), f_{0}=C, f_{1}=C \exp (-\eta / \zeta) \zeta^{-(k-1)}$. One then finds, at $s=0, f_{0} \cdot\left(f_{1} \circ \tau\right)=\frac{C^{2}}{(k-1) !} \eta^{k-1}+\cdots$, from which $C=((k-1) !)^{1 / 2}$. Note that $C$ does not depend on $S$.

The Rational Map. If one wants $(z I-B)^{-1}$ to be an endomorphism, then the formula $(8)$ is only $O(k, \mathbb{C})$ invariant. To compute $f(z)$ in a non-orthonormal frame, one has

$$
f(z)=W^{T} g^{T} \cdot g(z I-B)^{-1} W,
$$

where the columns of $g$ are the components of vectors of our frame with respect to an orthonormal one. One must thus compute the orthogonal structure of $E$ at $s=1$, with respect to our basis $v_{i}$. 
Referring to (16), $\left(v_{i}, v_{j}\right)=\left(\eta^{k-1}\right)$ term of $\sigma(a) v_{i} v_{j}$. As from [3, Prop. 4.5], this term is unique, it is given by the leading term of the Lagrange interpolation in $\eta$ of the values at $\eta_{h}, h=1, \ldots, k$ of $\sigma(a) v_{i} v_{j}$. Thus,

$$
\left(v_{i}, v_{j}\right)=\delta_{i j} \cdot b_{j} \cdot \prod_{m \neq j}\left(\eta_{j}-\eta_{m}\right)^{-1},
$$

where $b_{j}$ is the value at $\left(\eta_{j}, 0\right)$ of the representative $b^{0}(\eta, \zeta)$ over $\tilde{U}_{0}$ of $\sigma(a)$. One has $\left.g^{T} g=\operatorname{diag}\left(b_{j} \prod_{m \neq j}\left(\eta_{j}-\eta_{m}\right)^{-1}\right)\right)$; inserting $B, w$ and $g^{T} g$ into (20), $f$ has poles at $z=\eta_{j}$, with residues $(k-1) ! b_{j} \prod_{m \neq j}\left(\eta_{j}-\eta_{m}\right)^{-1}$. Thus, $f$ can be expressed in the following way, which extends to the case of $\eta_{i}$ not distinct. (We normalise by the $(k-1)$ !).

Theorem. Let $(\nabla, \Phi)$, an $\mathrm{SU}(2)$ monopole, have spectral curve $S$. Let $\sigma(a)$ be a normalised section of $L^{-2}$ over $S$, with representative functions $b^{i}$ over $\tilde{U}_{i} \cap S$, $b^{0}(\eta, \zeta)=\exp (-2 \eta / \zeta) \cdot b^{1}(\eta, \zeta)$. Let $g(\eta)$ be a monic polynomial of degree $k$ such that $S$, over $\zeta=0$, has equation $g(\eta)=0$. Let $h(\eta)$ be the polynomial of degree $(k-1)$ such that $h(\eta)=b^{0}(\eta, 0)$, over $S \cap\{\zeta=0\}$. Then $f(z)=h(z) / g(z)$ is a rational map associated to $(\nabla, \Phi)$.

We note again that the circle ambiguity in the definition of the rational map arises from the choice of normalised sections $\sigma(a)$.

\section{Interpretation in Terms of Monopoles}

There remains the problem of interpreting the above directly in terms of the monopole $(\nabla, \Phi)$. For this, one refers to [2]; for simplicity, we treat the case $\eta_{i}$ distinct.

The space $T \mathbb{P}_{1}$ is the space of oriented lines in $\mathbb{R}^{3}$; the subset $D=\{(\eta, 0) \mid \eta \in \mathbb{C}\}$ corresponds to parallel lines $\left(x_{1}(t), x_{2}(t), x_{3}(t)\right)=(\operatorname{Re} \eta, \operatorname{Im} \eta, t), t \in \mathbb{R}$. On the other hand, if $F$ is the 2-bundle over $\mathbb{R}^{3}$ on whose sections $\nabla, \Phi$ act in the standard representation, $S$ is the set of lines $C$ over which there is a section $s$ with:

$$
\left(\nabla_{t}-i \Phi\right) s=0, s \text { decays to zero at } t= \pm \infty .
$$

This then describes the poles of our rational map: they are given by $\left(x_{1}^{j}+i x_{2}^{j}\right)$ of the $k$ lines $C_{j}$ parallel to the $x_{3}$ axis over which there are sections satisfying (21).

These remains the interpretation of the residues, or equivalently of the $b_{i}$. Referring to [2, pp. 591-593], we can trivialise $F$ with sections that satisfy $\nabla_{t} s=0$ along the lines in $b$, in two different ways:

(a) So that the trivialisation is asymptotically flat along the plane $\left\{\left(x_{1}, x_{2}, t\right): x_{1}, x_{2} \in \mathbb{R}\right\}$ as $t \rightarrow+\infty$, at least in a finite region of the plane.

(b) So that the same is true, as $t \rightarrow-\infty$.

Furthermore, there are sections $A, B$ of $F$ over $\mathbb{R}^{3}, A$ constant with respect to the (a) trivialisation, $B$ constant with respect to the (b) trivialisation, $A, B$ of unit length, 
such that, normalising the solutions $s_{i}(t)$ of $(21)$ over $C_{i}$,

$$
\begin{aligned}
& \lim _{t \rightarrow+\infty} e^{t} t^{-k} S_{i}(t)-A=0, \\
& \lim _{t \rightarrow-\infty} e^{-t} t^{k} S_{i}(t)-b_{i} B=0 .
\end{aligned}
$$

(One must take into account the definition [2, p. $587 \mathrm{ff}$.] of $L$.) Thus the rational map is equivalent to giving:

- The $k$ lines $C_{i}$, parallel to the $x_{3}$-axis, over which there is a solution to (21).

- The "scattering data" $b_{i}$ over $C_{i}$, given by (22)

Donaldson's result then implies that each such choice of data determines a unique monopole; this data (allowing multiplicity in the $k$ lines, with a corresponding definition of the scattering data) classifies $\mathrm{SU}(2)$ monopoles; the data $\left(C_{i}, b_{i}\right)$ and $\left(C_{i}, z b_{i}\right), z$ a constant of norm one, correspond to the same monopole. This result is essentially the same as that of Atiyah [5] for hyperbolic monopoles.

\section{References}

1 Donaldson, S. K.: Nahm's equations and the classification of monopoles. Commun. Math. Phys. 96, 387-408 (1984)

2 Hitchin, N. J.: Monopoles and geodesics. Commun. Math. Phys. 83, 579-602 (1982)

3 Hitchin, N. J.: On the construction of monopoles. Commun. Math. Phys. 89, 145-190 (1983)

4 Nahm, W.: All self-dual multimonopoles for arbitrary gauge groups. (preprint) TH. 3172 CERN (1981)

5 Atiyah, M. F.: Magnetic monopoles in hyperbolic space, to appear in Proceedings of International Colloquium, Bombay

Communicated by A. Jaffe

Received December 20, 1984 